\title{
Intensity and reverberation as factors in the auditory perception of egocentric distance
}

\author{
DONALD H. MERSHON and L. EDWARD KING \\ North Carolina State University, Raleigh, North Carolina 27607
}

\begin{abstract}
Both auditory intensity and reverberation have previously been shown to be sufficient to produce systematically varying judgments of perceived distance when several values of the variable are presented repeatedly to the same observer. Such studies do not, however, indicate clearly whether these cues are functioning in an absolute or in a relative manner. An absolute cue to auditory distance would require that two groups presented with different values of the variable in question should report different values of perceived distance. Two experiments are reported in which intensity variation and reverberation are examined. The results showed that auditory intensity differences over a range of $20 \mathrm{~dB}$ did not serve as an absolute cue to auditory distance, but could serve as a strong cue to changes in such distance. A comparison of data obtained in a normally reverberatory setting (Experiment 1 ) and an anechoic chamber (Experiment 2) indicated that the state of reverberation could serve as an absolute cue, with greater reverberation being associated with greater perceived distances. Some of the results were discussed in terms of the possibility that the specific distance tendency (a concept developed to handle some phenomena in visual space perception) might have applicability to the study of auditory perceived distance as well.
\end{abstract}

Auditory space perception may conveniently be considered as having two complementary aspects: the perception of the direction of a source of sound (usually with respect to the midline of the body) and the perception of the distance of that sound source from the observer. The experiments and discussion of the present paper are concerned chiefly with the aspect of distance perception, and little mention will be made of those factors which influence perceived direction.

\section{Possible Factors in Auditory Distance Perception}

Several stimulus variables have been proposed as important factors in determining the apparent distances of sounds. Most of these suggested cues are related to the physical changes known to occur in an auditory stimulus when the source of the sound is moved physically farther away. A few seem to require some learning on the part of the observer, in order to be effective. Coleman (1963) has reviewed most of the earlier literature on this topic, and we will not repeat that discussion here. It is useful, however, to identify brietly the five factors most frequently studied (viz, intensity, reverberation, frequency spectrum, binaural differences, and the type of stimulus used) and indicate their possible contributions.

\footnotetext{
The authors would like to thank Curtis Brothers, Vince Lembo, and Sandra Womack for their help in running the experiments. The cooperation of the Department of Mechanical and Aerospace Engineering of North Carolina State University in lending the use of their anechoic chamber is gratefully acknowledged. Special thanks go to David O'Brien, of that department, for his assistance in setting up and calibrating the equipment of the second experiment. Requests for reprints of this paper should be sent to Donald $H$. Mershon. Department of Psychology, North Carolina State University, Raleigh, North Carolina 27607.
}

Intensity of a sound is often considered an obvious cue to distance, in that there is a well-known 6-dB loss in sound pressure for each doubling of distance in free space. It is not surprising, therefore, to find that judgments of apparent distance increase systematically, when the intensity of the sound at the ear is decreased (Gardner, 1969; von Békésy, 1949). Another common finding of such studies is that the judged distances are increasingly large underestimates of the physical (or simulated) auditory distances as the latter become very great. Coleman's (1962) results indicate that the systematic relationship between judged and physical distance with variation in intensity may require a learned familiarity with the range of intensities involved.

Reverberation has also been shown to be important, as has been recognized for many years by the broadcast industry (cf. Thurlow, 1971). In general, as sound sources move away from an observer in a natural indoor setting, the proportion of sound energy directly reaching the observer's ears decreases, while the proportion reaching the observer's ears after reflection (and delay) from surrounding surfaces increases. This may be called the reverberation cue to auditory distance. Its effectiveness in modifying judgments of auditory distance has been verified by von Békésy (1960) in a study with the effects of intensity and frequency spectrum. (see below) held constant. Systematic increases in reverberation led to increases in the judged distance of a sound.

Based upon both his own and earlier work, Coleman (1968) has suggested a dual role for the effects of frequency spectrum. Physical analyses of acoustic stimuli have shown that the high-frequency content of a complex auditory stimulus is reduced, relative to the low-frequency content, both for very 
close distances (under $1 \mathrm{~m}$ ) and for large distances (over $2-3 \mathrm{~m}$ ). This physical pattern of variation led Coleman (1968) to suggest that a decrease in the high-frequency content of a sound would result in decreasing the judged distance if the sound were perceived to be close, and increasing the judged distance if the sound were perceived to be farther away. He supported this notion with data which showed that a sound with an artifically reduced high-frequency content was judged to be farther away than the original "unfiltered" sound for all physical distances from 2.4 to $8.5 \mathrm{~m}$. It is, however, unfortunate that distances closer to the observer than $2.4 \mathrm{~m}$ were not used, in order to verify in the same experiment that the artificial reduction of high-frequency content would lead to the predicted reversal in effect, when the sounds were perceived to be close to the observer. To the extent that such dual perceptual effects are possible, they must depend upon some other factor(s) to produce an initial perception of whether the source is near or far. Only after such an initial value has been determined might it then be modified in a predictable fashion through the suggested frequency spectrum cue.

Several investigators have considered the possibility that binaural differences may influence the perception of auditory distance as well as being important in the perception of auditory direction (Coleman, 1963; Green, 1968; Hirsch, 1968; Holt \& Thurlow, 1969; Molino, 1973). The basis for such binaural contributions rests upon the uniqueness of the binaural characteristics of sounds which are both displaced from the midline and located within a few meters of the head. The results of the cited studies are contradictory concerning the actual effectiveness of the binaural cues to distance. Fortunately for the examination of other cue systems, it is agreed that the binaural effects (to the extent they do function) may be ignored for auditory stimuli presented radially along the midline.

As noted above, Coleman (1962) found that practice was necessary before his observers could use the intensity of an unfamiliar sound (white noise) as a basis for judging distance. The possible importance of familiarity is reflected in studies of auditory distance which have used speech, rather than tones or white noise, for stimuli (Gardner, 1969). Such studies support the idea that speech sounds can be judged in distance more readily than less familiar sounds. The quality of the speech may also be important, in that the distance to a whispering talker was underestimated, whereas the distance to a shouting talker was overestimated. Whether these latter effects are at least partially due to changes in frequency spectrum is difficult to evaluate.

\section{Methodological Considerations}

Before describing some experiments on the perception of auditory distance, it is necessary to discuss some methodological considerations which are important to such studies and which have frequently been ignored in the past. These considerations involve both the method to be used in measuring perceived distance and the choice of experimental design.

Many past investigators concerned with auditory distance have emphasized the ability (or lack of such) of observers to estimate the distance to a sound source rather than emphasizing the apparent distance to the sound. These are by no means the same aspect of the situation. The former, which has been termed a "cognitive" judgment in another context (cf. Mershon \& Gogel, 1975), implicitly suggests to the observer that more than the apparent distance should be taken into account. This emphasis is fine, if one is interested solely in obtaining accurate performance, but is inappropriate if one is concerned with perceptual variation per se.

The above emphasis is reflected experimentally in the frequently employed method of obtaining distance responses by using a set of midline speakers stretching away radially from the observer. For each sound presented, the observer is asked to identify the speaker from which the sound originated (cf. Coleman, 1962, 1968; Gardner, 1969; Molino, 1973). This technique has several limitations. One limitation is that such a procedure necessarily restricts the range of available answers which an observer may give, regardless of the apparent position of the sound. A second limitation is that the observer, particularly in making repeated judgments with the same set of speakers, may tend to distribute his responses over the available speakers, in order to equalize his choices of each. These are well-known problems with such category scaling techniques (Parducci, 1974).

In addition to the judgmental problems involved with the "speaker identification" method, there are perceptual ones. It is entirely reasonable that the presentation of a visual array may influence the perceptual distances of the auditory stimuli. An example of such an influence that has been found repeatedly is called the "proximity effect." This is the tendency on the part of the observer to hear the sound as if it were coming from the closest of the rationally possible sources (Gardner, 1968). Clearly, if one is interested in obtaining reports which more directly reflect the unmodified auditory perception of distance, it is crucial to eliminate extraneous visual cues as to the possible physical locations of the speaker or speakers used.

A somewhat different methodological criticism concerns the failure of many previous investigators to use an experimental design which can distinguish between what may be called egocentric and exocentric factors in auditory distance perception. Egocentric factors or absolute cues are those variables which directly affect the perception of the absolute distance between an observer and a source of sound. Such egocentric factors provide the basis for a perceptual 
scale of distance in terms of absolute values (feet, inches, meters, etc.). Exocentric factors or relative cues, on the other hand, are variables which, while they may indicate changes or differences in distance (i.e., "depth," the relative positions of sounds nearer or farther), do not themselves provide any absolute scale. Although absolute and relative cues usually interact in determining a perceptual result, they may involve some very different factors and should, for that reason, be kept conceptually distinct (cf. Gogel, 1968).

Generally, the appropriate test of whether a given variable is an important factor for the perception of egocentric distance requires that when different values of the cue are used for independent groups of observers, the average judgments of the two groups should differ significantly in the predicted direction. Certainly, the use of judgments from several different presentations to each observer (even if balanced or randomized) is not sufficient. What such data can demonstrate is whether changes in the tested factor may produce systematic changes in the perceived distance, i.e., whether the factor may be important as a cue to auditory depth. Any factor shown to be an effective absolute cue, of course, would be expected to provide the basis for making relative judgments of changes in distance as well. The reverse is not necessarily true; a factor may be a good relative cue to perceived depth without being effective in determining an absolute scale for perceived distance.

\section{Present Experiments}

The present experiments attempt to evaluate the effectiveness of auditory intensity and reverberation as factors in the perception of egocentric and exocentric distance in situations without extraneous visual cues and with instructions which request reports of "apparent" rather than judged (estimated) distance. Different combinations of intensity and reverberation are used as the initial presentation for separate groups of observers, so that comparison of the resulting "first-presentation" data will provide the required test of whether each factor can function as an absolute cue. Each group of observers is then presented with at least one additional stimulus for which the auditory intensity has been changed from that of the first presentation. Comparisons of the resulting "secondpresentation" data will indicate whether intensity changes can produce changes in auditory depth, even if intensity is ineffective in determining an absolute scale for auditory distance.

The principal difference between the two studies involves the acoustic situation in which they occurred. The first experiment took place in a generally reverberatory setting, the second in an anechoic chamber (a condition with little or no reverberation). In order to discuss the results of the two experiments jointly, we will first present the apparatus and procedural details for both studies.

\section{METHOD}

\section{Experiment 1}

Subjects. Observers for Experiment 1 were 80 introductory psychology students participating as a course requirement. It was requested that they have normal hearing, although no test was made to verify this. All were experimentally naive.

Apparatus. Stimuli were S-sec presentations of white noise. Noise was prerecorded from a Grason-Stadler (Model 901-B) noise generator and reproduced with a Sony (Model TC-350) tape deck connected to two General Electric FR-8 speakers through an amplifier and switching circuit. The switching circuit allowed the experimenter to present either of two intensity signals from either of the two speakers. Presentations were timed manually using a desk clock. The speakers were centered at ear level $(1.2 \mathrm{~m})$ inside a smooth surfaced tunnel, $1.8 \mathrm{~m}$ wide $\times 2.5 \mathrm{~m}$ high $\times 6.1 \mathrm{~m}$ long, constructed from the acoustically reflective panels used as backdrops in stage sets. The nearer speaker was located $2.74 \mathrm{~m}$ from the observer's position and the farther speaker at $5.49 \mathrm{~m}$ from the observer's position. Both ends of the tunnel were completely covered with opaque polyethelene curtains. The observer sat on a chair positioned just inside one end of the tunnel and facing the speakers. The tunnel was not lighted during the experiment.

Procedure. Observers were met at the door to the laboratory where they were given preliminary instructions and blindfolded before being led to the tunnel and seated. They were then given additional instructions about the task and provided with a large pad of paper and a pen with which to record their observations.

Approximately $1 \mathrm{~min}$ after the end of the instructions, two 5-sec bursts of white noise were presented from one of the two speakers. These noise presentations were separated by a 10-sec pause. Immediately following each presentation, the observer recorded the "apparent distance" from himself/herself to the sound "in feet or inches or in some combination of feet and inches." These records were made by writing on the pad while still blindfolded. Each record was later verified with the observer before he/she left the laboratory, to insure that no misinterpretations were made due to illegibility.

The two presentations to an observer differed in intensity. Noise was presented at an A-weighted sound level of either 60 or $50 \mathrm{~dB}$. All intensities were measured from the observer's head position with a General Radio permissible sound-level meter (Type 1565-A).

Half of the observers were presented with the sounds from the near speaker, half with the sounds from the far speaker. For each group, half of the observers heard the more intense sound first and half the less intense sound first. The second presentation of sound was always the other of the two possible intensities. Observers were assigned to conditions by the order in which they reported to the laboratory, and the running order of the conditions was counterbalanced for both speaker position and order of intensity presentation.

\section{Experiment 2}

Subjects. Observers for Experiment 2 were 80 introductory psychology sutdents participating as a course requirement. None had more than $5 \mathrm{~dB}$ hearing loss from $1-8 \mathrm{kHz}$, as measured by a Belltone audiometer (Model 12-C). All were experimentally naive and none had participated in Experiment 1.

Apparatus. Stimuli were 5-sec presentations of white noise produced directly from a Bruel and Kjaer noise generator (Type 1402) connected to a KLH (Model 17) speaker through an amplifier and switching circuit. The speaker was located in an anechoic chamber at either $2.74 \mathrm{~m}$ (near) or $5.49 \mathrm{~m}$ (far) from the observer's position in the chamber. The speaker was again at ear level and in the observer's midline. A chinrest attached to the observer's chair insured proper alignment. Presentations and pauses were timed automatically by an electrically driven cam timer. During the experiment, the lights in the chamber were off.

Procedure. Observers were met at the main door to the laboratory, as before, and blindfolded before being led to the anechoic chamber, seated, provided with the pad and pencil, and 
given final instructions. Approximately $1 \mathrm{~min}$ after the end of the instructions, six 5-sec bursts of white noise were presented from the speaker. Presentations were again separated by 10-sec pauses, during which the observer recorded the apparent distance to the immediately preceding sound.

A total of three different intensities were used for the experiment, although no individual observer was ever presented with more than two. When the near speaker location was used, noise was presented at an A-weighted sound level of either 65 or $55 \mathrm{~dB}$. When the far speaker location was used, noise was presented at either 55 or $45 \mathrm{~dB}$. All intensities were measured at the observer's head position with a Brüel and Kjaer audio frequency spectrometer (Type 2112) with calibrated microphone.

Half of the observers were presented with sounds from the near speaker, half with sounds from the far speaker. For each group, half of the observers were presented with the more intense of the two sounds on the first and the sixth trials and with the less intense of the two sounds on the second, third, fourth, and fifth trials. The other half of the observers were presented with the less intense sound on the first and sixth trials and with the more intense sound on the second, third, fourth, and fifth trials. Observers were assigned to conditions on the basis of their arrival at the laboratory, and the order of running the different conditions was counterbalanced.

\section{RESỦLTS}

Table 1 shows the data from the first and second presentations of Experiment 1 and from the first, second, and sixth presentations of Experiment 2. Since the results from judgments of perceived distance often tend to be skewed, both mean and median values are given and all statistical tests are nonparametric (Mann=Whitney $U$ tests).

\section{First Presentation Data}

Consider first the values given in the columns labeled "First Presentations." These represent the reports of the apparent distance $\left(D^{\prime}\right)$ between the observer and the first sound presented to him. To the extent that these values differ among groups of observers presented with different conditions, one may say that an absolute cue exists among the conditions used. For Experiment 1, there were no statistically significant differences in $D^{\prime}$ between the high- and low-intensity presentations for either the near or far speaker location. For Experiment 2, none of the conditions differed significantly from any of the others on first presentation, despite intensity variations of as much as $20 \mathrm{~dB}$ among the conditions. Taken as a whole, these results provide little support for the possibility that intensity can serve as an absolute cue to auditory distance.

All of the groups from the first experiment gave much larger judgments of $D^{\prime}$ on the first presentation than did any of the groups from the second experiment. This result holds for each of the four possible comparisons made between otherwise similar subgroups of the two experiments (all $p<.001$ ). The most reasonable explanation for this effect is that the extreme difference in the reverberation characteristics between the tunnel of Experiment 1 and the anechoic chamber of Experiment 2 was able to provide an absolute cue for auditory distance. In line with expectations, the virtually zero reverberation in the anechoic chamber led to a very small values of apparent distance. The greater reverberation in the tunnel resulted in larger values of apparent distance. This finding confirms those of von Békésy (1960), but extends them in showing that reverberation is not only

Table 1

Perceived Distance $\left(D^{\prime}\right)$ of a Sound from the Observer as a Function of Presentation, Speaker Location, and Sound Intensity

\begin{tabular}{|c|c|c|c|c|c|c|c|c|c|c|}
\hline & & \multicolumn{3}{|c|}{ First Presentations } & \multicolumn{3}{|c|}{ Second Presentations } & \multicolumn{3}{|c|}{ Sixth Presentations } \\
\hline & & Near & Far & Average & Near & Far & Average & Near & Far & Average \\
\hline & & \multicolumn{9}{|c|}{ Experiment 1} \\
\hline Low Intensity & $\begin{array}{l}\text { Mean } \\
\text { SD } \\
\text { Median }\end{array}$ & $\begin{array}{l}263.3 \\
146.0 \\
236.2\end{array}$ & $\begin{array}{l}289.0 \\
127.1 \\
304.8\end{array}$ & $\begin{array}{l}276.2 \\
136.6 \\
270.5\end{array}$ & $\begin{array}{l}282.9 \\
156.7 \\
259.1\end{array}$ & $\begin{array}{l}556.3 \\
400.5 \\
457.2\end{array}$ & $\begin{array}{l}419.6 \\
278.6 \\
358.2\end{array}$ & & & \\
\hline High Intensity & $\begin{array}{l}\text { Mean } \\
\text { SD } \\
\text { Median }\end{array}$ & $\begin{array}{l}242.3 \\
137.2 \\
182.9\end{array}$ & $\begin{array}{l}440.4 \\
275.8 \\
335.3\end{array}$ & $\begin{array}{l}341.4 \\
206.5 \\
259.1\end{array}$ & $\begin{array}{l}193.9 \\
110.0 \\
167.6\end{array}$ & $\begin{array}{l}202.7 \\
125.3 \\
182.9\end{array}$ & $\begin{array}{l}198.3 \\
117.6 \\
175.2\end{array}$ & & & \\
\hline \multirow[t]{2}{*}{ Average } & $\begin{array}{l}\text { Mean } \\
\text { SD } \\
\text { Median }\end{array}$ & $\begin{array}{l}252.8 \\
141.6 \\
209.6\end{array}$ & $\begin{array}{l}364.7 \\
201.4 \\
320.0\end{array}$ & & $\begin{array}{l}238.4 \\
133.4 \\
213.4\end{array}$ & $\begin{array}{l}379.5 \\
262.9 \\
320.0\end{array}$ & & & & \\
\hline & & \multicolumn{9}{|c|}{ Experiment 2} \\
\hline Low Intensity & $\begin{array}{l}\text { Mean } \\
\text { SD } \\
\text { Median }\end{array}$ & $\begin{array}{l}46.9 \\
37.8 \\
30.5\end{array}$ & $\begin{array}{l}56.1 \\
46.3 \\
45.7\end{array}$ & $\begin{array}{l}51.5 \\
42.0 \\
38.1\end{array}$ & $\begin{array}{l}75.6 \\
82.3 \\
45.7\end{array}$ & $\begin{array}{l}64.6 \\
62.2 \\
45.7\end{array}$ & $\begin{array}{l}70.1 \\
72.2 \\
45.7\end{array}$ & $\begin{array}{l}56.7 \\
61.9 \\
38.1\end{array}$ & $\begin{array}{l}75.0 \\
57.0 \\
76.2\end{array}$ & $\begin{array}{l}65.8 \\
59.4 \\
57.2\end{array}$ \\
\hline High Intensity & $\begin{array}{l}\text { Mean } \\
\text { SD } \\
\text { Median }\end{array}$ & $\begin{array}{l}57.9 \\
75.3 \\
30.5\end{array}$ & $\begin{array}{l}35.7 \\
32.9 \\
30.5\end{array}$ & $\begin{array}{l}46.8 \\
54.1 \\
30.5\end{array}$ & $\begin{array}{l}29.6 \\
23.5 \\
25.6\end{array}$ & $\begin{array}{l}34.4 \\
28.3 \\
22.9\end{array}$ & $\begin{array}{l}32.0 \\
25.9 \\
24.2\end{array}$ & $\begin{array}{l}31.4 \\
36.9 \\
15.2\end{array}$ & $\begin{array}{l}27.4 \\
32.3 \\
20.4\end{array}$ & $\begin{array}{l}29.4 \\
34.6 \\
17.8\end{array}$ \\
\hline Average & $\begin{array}{l}\text { Mean } \\
\text { SD } \\
\text { Median }\end{array}$ & $\begin{array}{l}52.4 \\
56.6 \\
30.5\end{array}$ & $\begin{array}{l}45.9 \\
39.6 \\
38.1\end{array}$ & & $\begin{array}{l}52.6 \\
52.9 \\
35.6\end{array}$ & $\begin{array}{l}49.5 \\
45.2 \\
34.3\end{array}$ & & $\begin{array}{l}44.0 \\
49.4 \\
26.6\end{array}$ & $\begin{array}{l}51.2 \\
44.6 \\
48.3\end{array}$ & \\
\hline
\end{tabular}

Note-Results from both experiments. All values converted to centimeters. 
a cue to changes in distance, but is an absolute cue for egocentric distance as well.

Such an absolute reverberation cue to distance may also be observed within Experiment 1. Thus, one may note that the sounds presented from the near speaker on first presentation were perceived as being closer to the observer than the sounds presented from the far speaker. This difference was statistically significant for the more intense sounds ( $p<.05$ ), but not for the less intense sounds. Under the tunnel conditions, of course, one would expect the magnitude of the reverberation cue to increase with an increase in physical distance, leading to larger values of apparent distance. One might also expect to have this effect be more clear for higher intensity presentations. Under the anechoic chamber conditions, however, no changes in this absolute cue should be expected as a function of the change in physical distance from 2.74 to $5.49 \mathrm{~m}$. And, as described above, no differences in perceived distance occurred among the "firstpresentation" data of Experiment 2.

\section{Later Presentation Data}

Consider now the values in the columns labeled "Second Presentations." For every group of observers, the report of perceived distance changed in the appropriate direction when the intensity changed between the first and second presentations (i.e., the perceived distance increased when the intensity was reduced and decreased when the intensity was raised). The differences between these second presentation values were all significant $(p<.05)$, except for that between the high- and low-intensity presentations from the near speaker for Experiment 1 . This overall pattern of results indicates that the intensity change in an auditory stimulus is a relatively strong cue for change in perceived distance (i.e., it is a relative cue to auditory depth).

There were only two presentations of sound in Experiment 1, but there were a total of six presentations in Experiment 2. Generally the reported distances for the third through the fifth presentations of Experiment 2 did not differ in any systematic way from the results for the second presentation. Hence, these data have not been included in Table 1. The lack of reported change, however, might be attributable to a cognitive bias based upon the observer's recognition that the sounds did not differ in intensity among these presentations and a preference not to report small or gradual changes in distance. It is interesting, therefore, to consider what predictions might be made for the $\mathrm{D}^{\prime}$ judgment for the sixth presentation, if the perceived distance were or were not varying between the second and fifth presentation.

The intensity on the sixth presentation was the same as on the first presentation for each observer. If the perceived distance on the fifth presentation were the same as for the second presentation, it would indicate a long-lasting effect of the relative intensity cue between the first and second presentations. Under such conditions, the reversal to the original intensity on the sixth presentation should simply produce reports of $\mathrm{D}^{\prime}$, which again did not differ on the basis of intensity from each other (or from the $D^{\prime}$ values of the first presentations). If, on the other hand, the relative intensity cue had actually "decayed" in effect over repeated presentations, despite the constant reported values of $\mathrm{D}^{\prime}$, then the return on the sixth presentation to the original intensity should produce another relative cue. Under such conditions, the $D^{\prime}$ values from the sixth presentations should show a clear difference due to intensity, even though the observers involved failed to show such a difference on the first presentations of the same intensities.

The actual results from the sixth presentations of Experiment 2 support the second possibility. Observers presented with the high intensity on the sixth presentation reported the apparent distance to be significantly less than did the observers presented with the low intensity $(\mathrm{p}<.05$ and $\mathrm{p}<.01$ for the near and far speaker locations, respectively). This result is strong additional support for the earlier conclusion that, although a change in auditory intensity may be a good relative cue for auditory depth, it is ineffective as an absolute cue when auditory stimuli are presented either initially or after a period without change. Rather, we must look to other factors such as reverberation for the determination of absolute auditory distance.

\section{DISCUSSION}

The two experiments described above indicated that the intensity of a sound can serve only as a relative cue to changes in egocentric auditory distance. In contrast to this result, the state of auditory reverberation was found to serve as an absolute cue to egocentric distance. The following discussion will examine the validity and implications of these results.

\section{Reverberation as an Absolute Cue}

The conclusion that reverberation is an absolute cue to egocentric auditory distance is based upon two aspects of the data. The first involves the large differences in reported $D^{\prime}$ between Experiments 1 and 2 for the first presentation judgments. The second involves the differences in $\mathrm{D}^{\prime}$ between the two speaker locations which were found for the tunnel conditions of Experiment 1 but not for the anechoic chamber conditions of Experiment 2. It must be emphasized here that the criterion for deciding whether a factor is an absolute cue requires only that there be systematic differences between independent groups of observers for whom the factor assumes different values. Thus. 
the fact that most of the reported distances in the two experiments are generally not veridical is not crucial. ${ }^{1}$

A more serious concern arises from the fact that some of the conclusions about the importance of reverberation are based upon a comparison across experiments which differed slightly in apparatus and procedure. The differences in intensity used are not important, since the values of Experiment 2 encompass those of Experiment 1 and intensity was not shown to be an absolute cue under any of the conditions. The quality of the audio equipment, however, was somewhat superior in the second experiment, and that is worth additional consideration. It is likely, for example, that the improved equipment in Experiment 2 led to proportionately increased high-frequency content in the auditory stimuli of that experiment, relative to those of Experiment 1. Such an increase in high-frequency content should lead to larger judgments of $D^{\prime}$, if the source were perceived to be as close to the observer as they were in the second experiment [cf. Coleman's (1968) dual-effect hypothesis]. Thus, one would expect that the observed differences in perceived distance between the first presentations of the two experiments are, if anything, underestimations of the difference due to the differing values of reverberation.

\section{An Auditory Specific Distance Tendency?}

One factor not previously considered with respect to auditory distance perception is the possible influence of the perceptual organizational factor known as the specific distance tendency (SDT). This is the tendency for objects presented under conditions of reduced information for egocentric distance to appear at a relatively near distance of approximately $2 \mathrm{~m}$ (Gogel, $1969,1973 a, b)$. Although to date the SDT has been demonstrated only as a factor in the visual perception of egocentric distance, it is not intrinsically limited to the visual modality. Rather, it may represent a general perceptual tendency independent of the spatial modality involved in a given situation.

If the SDT were effective as a factor in auditory space perception, it would be expected to determine the results most clearly when effective cues to egocentric distance were reduced. Since complete removal is either difficult or impossible to achieve, one needs to consider the combined effects of the SDT and those other factors which are present in the situation. The perceptual distance expected from this combination may be termed the egocentric reference distance (cf. Gogel, 1972). In an auditory experiment, then, any auditory stimulus presented in isolation (i.e., temporally separated from other different auditory stimuli) should appear at this reference distance. Subsequent auditory stimuli may then appear displaced in distance, if an effective relative cue exists between the two stimuli.

In the present experiments, the SDT would be modified by the state of reverberation (a demonstratedly effective cue) existing for each condition. The resulting reference distance would lead to a constant value of $D^{\prime}$ for all presentations involving the given condition of reverberation. Thus, the reference distance would be an important determiner of the perceived distance both for the first presentations and also increasingly for later presentations, when such presentations were temporally separated from the previous relative intensity cue (by repetition of a constant intensity). The reversal in $D^{\prime}$ for the sixth presentations would thus be due to the new relative cue being superimposed upon sounds which had returned to this reference distance. This interpretation is entirely congruent with the facts of the experiments and is the one favored by the authors, but it is important to state that it is not the only possible interpretation to be made.

Given that reverberation can serve as an absolute cue to distance, the differences found in the experiments might be attributable to a reference distance determined solely and directly by the specific value of reverberation which existed for a particular condition. The notion of a specific distance tendency as a general perceptual factor in auditory distance perception would be superfluous.

The existence of the specific distance tendency for auditory space depends for its viability on a demonstration that close physical distances are overestimated and far physical distances are underestimated, when systematic variation in an effective absolute cue (like reverberation) is allowed. That is, the range of perceived distances must be "collapsed" relative to the range of physical distances used. Such a collapse of the range of apparent distances on first presentations is suggested by the results of Coleman (1962) and for repeated presentations by those of Coleman (1968), but those experiments both used the criticized method of obtaining judgments with a simultaneously visible array of speakers. Further studies on the question are currently in progress.

\section{A Practical Implication}

The possible significance of this research to one practical problem should be pointed out. Attempts have been made to construct sonar-like devices for the blind which generate inaudible signals (either ultrasonic or infrared) and capture the echoes of those signals which are reflected from objects in the surrounding environment. The device then converts the information in the echoes into auditory signals which the wearer may learn to use in his personal navigation (Mims, 1973; Thornton, 1971).

The results of our experiments would suggest the possibility that the use of such devices might be more readily learned if the information concerning the egocentric distances of objects were presented to the wearer in the form of variations in reverberation, 
rather than in the commonly employed form of pitch variations. This suggestion is based upon the finding that variations in reverberation do result in systematic differences in absolute perceived distance. We expect that learning to "rescale" (calibrate) one's responses to this kind of cue variation would be easier than trying to learn to recognize and cognitively interpret variations from a cue which does not inherently involve absolute perceptual values for distance in the first place. The use of a "natural" cue system might also lessen the problem of distracting the wearer from his ambient auditory environment by being more similar to the effective auditory distance cues from environmental objects which normally emit sounds.

\section{REFERENCES}

Coleman. P. D. Failure to localize the source distance of an unfamiliar sound. Joumal of the Acoustical Society of America, 1962, 34. 345-346.

Coleman, P. D. An analysis of cues to auditory depth perception in free space. Psychological Bulletin, 1963, 60, 302-315.

Coleman, P. D. Dual role of frequency spectrum in determination of auditory distance. Journal of the Acoustical Society of America. 1968, 44, 631-632.

GARDNER, M. B. Proximity image effect in sound localization. Journal of the Acoustical Society of America, 1968, 43, 163.

GARDNER, M. B. Distance estimation of $0^{\circ}$ or apparent $0^{\circ}$-oriented speech signals in anechoic space Joumal of the Acoustical Society of America, 1969, 45, 47-53.

GOGEL, $W$. C. The measurement of perceived size and distance. In W. D. Neff (Ed.), Contributions to sensory physiology (Vol. 3). New York: Academic Press, 1968.

GoGEL, W. C. The sensing of retinal size. Vision Research, 1969, 9. 1079-1094.

Gogel, W. C. Scalar perceptions with binocular cues of distance. American Journal of Psychology, 1972. 85, 477.497.

GoGEl. W. C. The organization of perceived space. I. Perceptual interactions. Psychologische Forschung, 1973, 36, 195-221. (a)

GoGEL, W. C. The organization of perceived space. II. Consequences of perceptual interactions. Psychologische Forschung, 1973, 36. 223-247. (b)
GREENE, D. C. Comments on "Perception of the range of a sound source of unknown strength." Journal of the A coustical Society of America, 1968, 44, 634.

Hirsch, H. R. Perception of the range of a sound source of unk nown strength. Journal of the Acoustical Society of America, 1968, 43, 373-374.

Holt, R. E., \& Thurlow, W. R. Subject orientation and judgment of distance of a sound source. Joumal of the Acoustical Society of America, 1969, 46, 1584-1585.

Mershon, D. H., \& Gogel, W. C. Failure of familiar size to determine a metric for visually perceived distance. Perception \& Psychophysics, 1975, 17, 101-106.

Mims, F. M. Sensory aids for blind persons. The New Outlook for the Blind, 1973, 67, 407-414.

Molino, J. Perceiving the range of a sound source when the direction is known. Journal of the Acoustical Society of America, 1973, 53, 1301-1304:

PArducci, A. Contextual effects: A range-frequency analysis. In E. C. Carterette and M. P. Friedman (Eds.), Handbook of perception (Vol. 2), Psychophysical judgment and measurement. New York: Academic Press, 1974.

Thornton, $W$. The binaural sensor as a mobility aid. The New Outlook for the Blind, 1971, 65, 324-326.

Thurlow. W. R. Audition. In J. W. Kling \& L. A. Riggs (Eds.), Woodworth and Schlosberg's Experimental psychology (3rd ed.). New York: Holt, Rinehart \& Winston, 1971.

VoN BÉKÉsY, G. The moon illusion and similar auditory phenomena. American Journal of Psychology, 1949, 62, 540-552.

voN BÉkÉsy, G. The moon illusion and similar auditory phenomena. American Journal of Psychology, 1949, 62, 540-552.

\section{NOTE}

1. Indeed, some of the "accuracy" shown in previous work on auditory distance perception may be spurious, the result of presenting a simultaneous array of alternative speakers as choices (see introduction). The observer is thus able to use his genuinely varying perceptions of egocentric distance to choose (cognitively) a relatively near or far source.

(Received for publication May 5, 1975; revision received July 30,1975 .) 